\title{
REMOÇÃO DO CORANTE AZUL DE METILENO NO TRATAMENTO DE EFLUENTES POR ADSORÇÃO EM BAGAÇO DE CANA DE AÇÚCAR
}

\author{
I. R. JORGE ${ }^{1}$, F. P. TAVARES ${ }^{1}$, K. G. SANTOS ${ }^{1 *}$ \\ ${ }^{1}$ Universidade Federal do Triângulo Mineiro, Departamento de Engenharia Química \\ e-mail: kassiagsantos@gmail.com
}

\begin{abstract}
RESUMO
As indústrias têxteis estão crescendo cada vez mais, hoje em dia, cerca de 10.000 tipos diferentes de corantes e pigmentos são utilizados industrialmente e a estimativa de corantes descartados junto aos despejos dos efluentes

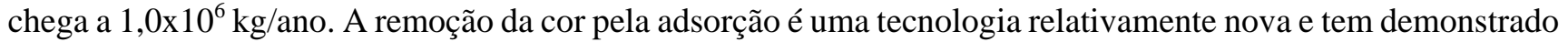
bastante eficiência, visto que é uma tecnologia barata e de simples operação. No entanto, devido ao elevado custo dos adsorventes comerciais, materiais alternativos que unam uma boa capacidade de adsorção com um baixo custo, estão sendo estudados. O bagaço de cana de açúcar se encontra de maneira abundante como o maior resíduo da indústria sucroalcooleira do país, esse fator juntamente com suas características estruturais, o torna um material adsorvente em potencial. O presente trabalho estudou a capacidade do BC em adsorver o corante AM, através do levantamento de dados experimentais foram obtidas as isotermas de equilíbrio, para avaliar o desempenho do adsorvente, a cinética do processo determinando o tempo necessário para chegar ao equilíbrio, e a dinâmica de adsorção em uma coluna de leito fixo. O BC apresentou um elevado potencial para a adsorção do AM, seguindo o modelo de Sips a cinética de adsorção de pseudo-segunda ordem. As curvas de ruptura apresentaram ajustes satisfatórios a todos os modelos estudados, e o que mais se destacou foi o modelo de Yan.
\end{abstract}

Palavras-chave: Adsorção, Bagaço de Cana-de-Açúcar, Leito Fixo.

\section{INTRODUÇÃO}

Atualmente, as indústrias têxteis estão crescendo cada vez mais, e cerca de 10.000 tipos diferentes de corantes e pigmentos são utilizados industrialmente, gerando um consumo anual de 700.000 toneladas no mundo e 26.500 toneladas no Brasil. Como resultado a esses dados, a estimativa de corantes descartados junto aos despejos dos efluentes da indústria têxtil chega a $1,0 \times 10^{6}$ kg/ano (GUARATINI; ZANONI, 2000).

A maior parte dos corantes fabricados destina-se a indústria têxtil, em seguida vêm às indústrias de artesanatos de couro e de papel, indústrias alimentícias, cosméticos, tintas e plásticos, mostrando que esse tipo de produto tem aplicação universal e justificando tamanha diversidade, uma vez que cada tipo de fibra requer corantes com características próprias e bem definidas (GUARATINI; ZANONI, 2000).

Uma vez aplicado ao tecido, o corante deve resistir a agentes responsáveis pelo desbotamento como a lavagem, o suor, a água clorada, a luz, entre outros (SILVA, 2005). Portanto, para garantir todas essas propriedades, a característica fundamental dos corantes é a estabilidade de suas moléculas, o que faz com que os efluentes descartados se tornem difíceis de serem descoloridos. Segundo Oliveira (2012), este tipo de contaminação confere características visuais e organolépticas diferentes dos sistemas de água potável, o que impossibilita seu consumo e o seu uso em geral.

Conforme a Resolução CONAMA n³57/05 (MINISTÉRIO DO MEIO 
AMBIENTE, 2006) complementar a Resolução CONAMA n²0/86., o efluente descartado não pode alterar a classificação do corpo receptor, e de acordo com a classificação e o nível que este se encontra, a coloração no efluente deve ser desde virtualmente ausente até o máximo de $75 \mathrm{mg}$ PT-Co/L.

Dentro desse contexto, o desenvolvimento de tecnologias adequadas para o tratamento desse tipo de efluente tem ganhado destaque.

Oliveira (2012) cita que as principais técnicas disponíveis na literatura para descoloração das águas de rejeito envolvem principalmente os processos de adsorção, precipitação, degradação química, eletroquímica, fotoquímica, biodegradação, entre outros.

Contudo, a aplicação do processo de adsorção vem sendo amplamente estudada para a remoção de corantes de águas residuais, uma vez que tem sido considerada superior a outras técnicas, por envolver menores custos, simplicidade de operação e apresentar alta eficiência, além de não formar intermediários, e sendo dependente apenas de fatores físicoquímicos, como a área superficial do adsorvente, tamanho da partícula, características estruturais e morfológicas, interação adsorvente/corante, $\mathrm{pH}$, temperatura e tempo de contato (SCHIMELL, 2008).

Segundo McCabe et al. (2014), o processo de adsorção apresenta-se como um fenômeno físico de transferência de massa que ocorre nas interfaces do sistema fluido-sólido, e que consiste na adsorção seletiva de alguns componentes da fase fluida na superfície sólida. O elemento que fica retido na parte sólida é denominado de adsorbato, e o sólido que mantém o adsorbato na sua superfície é denominado de adsorvente. Alguns exemplos dos processos industriais que tem aplicação da adsorção são: recuperação de vapores de solventes valiosos, branqueamento das soluções de açúcar, óleos vegetais e minerais, secagem de gases, remoção de contaminantes em efluentes industriais, dentre outras.

Um corante amplamente utilizado na indústria têxtil é o azul de metileno (AM), classificado como um corante do tipo básico, possuindo estrutura molecular aromática heterocíclica, com fórmula química $\mathrm{C}_{16} \mathrm{H}_{18} \mathrm{~N}_{3} \mathrm{SCl}$ e massa molar de $319,8 \mathrm{~g} / \mathrm{mol}$. Conforme citado por Oliveira (2012), é considerado um composto modelo para o estudo de remoção de contaminantes em soluções aquosas, sendo muito utilizado em testes de adsorção.

Um fator limitante para o processo de adsorção é que normalmente, os adsorventes comerciais são caros e de difícil regeneração, o que incentiva a busca por adsorventes alternativos para tornar o processo mais eficaz e atrativo economicamente. Dentro desse quadro se encontra o bagaço de cana-de-açúcar (BC), o qual é um material fibroso, e formado por celulose, hemicelulose e lignina.

A cana-de-açúcar se transformou em uma das principais culturas da economia brasileira, tornado o país o maior produtor de cana, e também o primeiro do mundo na produção de açúcar e etanol. Assim, devido a crescente capacidade dessas indústrias, quantidades cada vez maiores de BC estão sendo produzidas, tornando-o o maior resíduo agroindustrial do país, sendo gerado anualmente cerca de 5 a 12 milhões de toneladas/ano (MAPA, 2015).

Alternativas vêm sendo estudadas como forma de realocar todo o resíduo gerado de maneira adequada. Dentre estas, destaca-se, a aplicação de biomassas em processos de adsorção, apresentando elevado potencial na adsorção de poluentes.

A adsorção em coluna de leito fixo é um dos procedimentos mais utilizados industrialmente na área de processos químicos, devido a sua facilidade de construção e controle, e por apresentar resultados eficientes. Das curvas de ruptura são obtidas várias características das colunas como: o volume de 


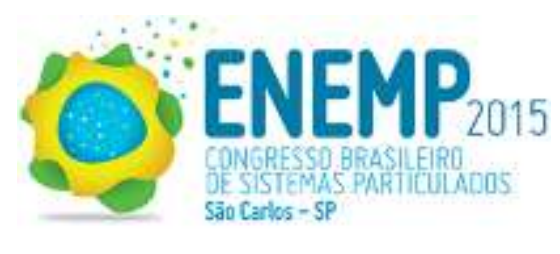

ruptura, a quantidade adsorvida na ruptura, a capacidade fracional, a altura da zona de transferência de massa (ZTM) e a velocidade de adsorção (ZANG et al., 2011).

Contudo, a dinâmica de adsorção que ocorre no leito fixo é complexa e de difícil modelagem devido aos vários fenômenos que ocorrem simultaneamente à transferência de massa, além da variação dos perfis de concentração nas fases sólida e líquida com o espaço e tempo, não sendo possível a operação em estado estacionário (ZANG et al., 2011).

$\mathrm{O}$ estudo do tratamento de efluentes têxteis através da adsorção do corante azul de metileno utilizando bagaço de cana-de açúcar é o tema do presente trabalho, o qual irá estudar o equilíbrio e a cinética de adsorção do sistema em temperatura ambiente. Esses dados foram empregados no projeto de um adsorvedor de leito fixo, no qual foi avaliada a eficiência de remoção do corante com o tempo de operação a fim de determinar o tempo de ruptura.

\section{METODOLOGIA}

\subsection{Caracterização do material adsorvente}

$\mathrm{O}$ BC foi primeiramente peneirado para obter uma granulometria menor que $2 \mathrm{~mm}$, e logo em seguida, o material foi lavado com água corrente e seco em estufa a $90^{\circ} \mathrm{C}$ por 24 horas, a fim de retirar algumas impurezas e evitar que o processo de adsorção fosse prejudicado. Ao fim, o BC foi armazenado em embalagens plásticas a temperatura ambiente.

\subsection{Isotermas de Adsorção}

Os experimentos de adsorção foram realizados em batelada com béqueres de 1,5 $\mathrm{L}$ e condição ambiente, sob agitação constante por um período de 24 horas. Após o fim dos experimentos as amostras foram filtradas com tecido de nylon para serigrafia e logo em seguida, a concentração da solução remanescente foi determinada pela leitura da absorbância no espectrofotômetro de UV-VIS, no comprimento de $664 \mathrm{~nm}$.
Para a construção da isoterma de adsorção utilizou soluções de diferentes com concentrações de AM (2, 5, 10, 20, 30, 40, 50, $100,200,300,400$ e $500 \mathrm{mg} / \mathrm{L}$ ) com volume fixo de $500 \mathrm{~mL}$ e, uma quantidade fixa de material adsorvente, de 2,5 g.

Com os dados obtidos, foi possível calcular a quantidade de AM adsorvido pelo BC através da Equação 1, e fazer o ajuste aos modelos, citados anteriormente na Tabela 1.

$q_{e}=\frac{V\left(C_{0}-C_{e}\right)}{m}$

onde $q_{e}$ é a quantidade de AM adsorvido pelo $\mathrm{BC}$ no equilíbrio $(\mathrm{mg} / \mathrm{g}) ; C_{0}$ é a concentração inicial de $\mathrm{AM}(\mathrm{mg} / \mathrm{L}) ; C_{e}$ é a concentração de AM no equilíbrio (mg/L); $V$ é o volume da solução (L); e $m$ é a massa do adsorvente (g).

A obtenção dos dados de equilíbrio e a descrição desses por modelos matemáticos, indicam como será o processo efetivamente, se a purificação requerida pode ser obtida, a estimativa da quantidade máxima de soluto que o adsorvente adsorverá $\left(q_{e}\right)$ e informações que determinam se o adsorvente pode ser economicamente viável para a purificação do líquido (SCHIMMEL, 2008).

Os modelos mais utilizados estão apresentados na Tabela 1.

\subsection{Cinética de Adsorção}

A cinética de adsorção possui fundamental importância para o projeto de sistemas de tratamento de efluentes, pois descreve a velocidade com que as moléculas do adsorbato são adsorvidas pelo material adsorvente. Tal parâmetro depende das características físico-químicas do adsorbato, adsorvente, e da solução, como o tamanho das moléculas de adsorbato, distribuição e tamanho dos poros, e da variabilidade do efluente (SCHIMMEL, 2008). Além disso, a cinética de adsorção pode ser representada por modelos, como os apresentados na Tabela 2. 
Tabela 1 - Modelos de Isotermas de Adsorção.

\begin{tabular}{ll}
\hline Modelos & Equação \\
\hline Langmuir & $q_{e}=\frac{q_{\text {máx }} b_{L} C_{e}}{1+b_{L} C_{e}}$ \\
Freundilch & $q_{e}=k_{F} C_{e}{ }^{{ }^{2}}$ \\
Sips & $q_{e}=\frac{q_{\text {máx }}\left(k_{s} C_{e}\right)^{n_{s}}}{1+\left(k_{s} C_{e}\right)^{n_{s}}}$ \\
Temkin & $q_{e}=\beta \ln \left(k_{T e} C_{e}\right)$ \\
Tóth & $q_{e}=\frac{q_{\text {máx }} C_{e}}{\left(1 / k_{T}+C_{e}{ }^{n_{T}}\right)^{1 / n_{T}}}$ \\
Radke e & $q_{e}=\frac{q_{\text {máx } b_{r a d} C_{e}}^{\left(1+b_{r a d} C_{e}\right)^{n_{r a d}}}}{\text { Praunsnitz }}$ \\
Redlich e & $q_{e}=\frac{k_{R P} C_{e}}{1+\alpha_{R P} C_{e}^{g}}$ \\
Peterson &
\end{tabular}

Fonte: Adaptado de Colombo (2013).

Tabela 2 - Modelos Cinéticos de Adsorção

\begin{tabular}{ll}
\hline Modelo & Equação \\
\hline Pseudo $1^{\mathrm{a}}$ Ordem & $q_{t}=q_{e 1}\left[1-\exp \left(-k_{1} t\right)\right]$ \\
Pseudo $2^{\mathrm{a}}$ Ordem & $q_{t}=\frac{k_{2} q_{e 2}{ }^{2} t}{1+q_{e 2} k_{2} t}$
\end{tabular}

Fonte: Adaptado Schimell (2008).

O levantamento da curva cinética de adsorção foi realizado com soluções de $1 \mathrm{~L}$ do efluente simulado, com concentrações de 50 e $200 \mathrm{mg} / \mathrm{L}$, definidas a partir do estudo da isoterma de adsorção, e uma quantidade fixa de $5 \mathrm{~g}$ de adsorvente, retirando-se 24 alíquotas em intervalos de tempo pré-estabelecidos.

A quantidade de AM adsorvida pelo BC em cada intervalo de tempo foi obtida utilizando a Equação 2, e a análises dos dados foi realizada através do ajuste aos modelos cinéticos citados anteriormente na Tabela 2.

$q_{t}=\frac{V\left(C_{0}-C_{t}\right)}{m}$

Em que $q_{t}$ é a quantidade de AM adsorvido pelo BC no tempo $t(\mathrm{mg} / \mathrm{g}) ; C_{0}$ é a concentração inicial da solução de AM (mg/L); $C_{t}$ é a concentração de AM no tempo $t(\mathrm{mg} / \mathrm{L})$;
$V$ é o volume da solução (L); e $m$ é a massa do adsorvente (g).

\subsection{Adsorção em Leito Fixo}

Para realizar os estudos de adsorção em processo contínuo, o equipamento de leito fixo foi construído, como mostra a Figura 1 a partir de tubo de PVC de $50 \mathrm{~mm}$ de diâmetro interno e altura de $40 \mathrm{~cm}$, contendo dois recipientes com capacidade de 20 litros, um para alimentação da solução de corante e o outro para a coleta do efluente tratado, e mangueiras de silicone na entrada e na saída para conectar a coluna aos reservatórios.

Figura 1 - Unidade Experimental.

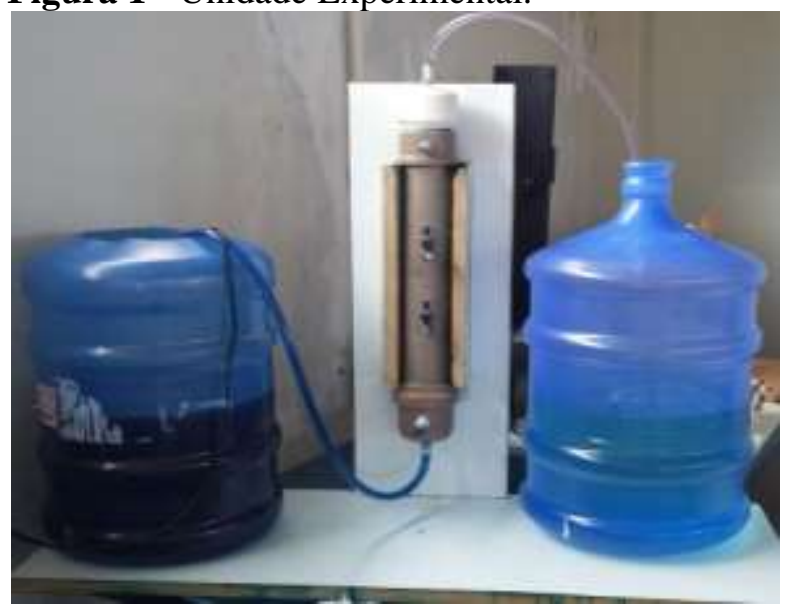

Fonte: Da Autora (2015).

A alimentação foi feita com o auxílio de uma bomba de circulação pela parte inferior do equipamento, adotando um fluxo de sentido ascendente, o qual fluiu através do leito de partículas até a parte superior da coluna.

$\mathrm{O}$ equipamento foi empacotado com BC e alimentado com uma solução de AM, e à saída da coluna foram recolhidas amostras em diferentes intervalos de tempo, a fim de obter dados para construir a curva de ruptura, as quais foram analisadas através da leitura de absorbância no espectrofotômetro.

Foram obtidas três curvas de ruptura. A Tabela 3 apresenta as condições experimentais estudadas no leito fixo. Todos os experimentos foram realizados à temperatura ambiente e, 
com a altura da coluna mantida constante, preenchendo todo o interior do equipamento com o material adsorvente.

Tabela 3 - Condições operacionais da adsorção do AM em leito fixo empacotado com BC.

\begin{tabular}{lccc}
\hline Experimento & $\begin{array}{c}\mathrm{C}_{0} \\
{[\mathrm{mg} / \mathrm{L}]}\end{array}$ & $\begin{array}{c}\mathrm{Q} \\
{[\mathrm{mL} / \mathrm{mi}]}\end{array}$ & $\begin{array}{c}\mathrm{m} \\
{[\mathrm{g}]}\end{array}$ \\
\hline Condição 1 & 50 & 219,6 & 116 \\
Condição 2 & 200 & 77,0 & 93,0 \\
Condição 3 & 200 & 202,0 & 104,4 \\
\hline
\end{tabular}

Devido a isso, a análise da curva de ruptura pode ser complementada através do ajuste dos dados aos modelos existentes na literatura, nesse trabalho foram utilizados os modelos disponíveis na Tabela 4. A partir dos dados experimentais obtidos, foi possível construir a curva de ruptura do leito fixo para as diferentes condições estudadas, e ajustar os resultados aos modelos de Thomas, Yoon \& Nelson, Yan e Wolborska.

Tabela 4 - Modelos de Curva de Ruptura

\begin{tabular}{cl}
\hline Modelos & Equação \\
Thomas & $\frac{C}{C_{0}}=\frac{1}{1+\exp \left[\frac{K_{T h}}{Q}\left(q_{T h} m-C_{0} Q t\right)\right]}$ \\
Yoon \& & $\frac{C}{C_{0}}=\frac{1}{1+\exp \left[k_{Y N}\left(\frac{q_{Y N}}{C_{0} Q}-t\right)\right]}$ \\
Nelson & $\frac{1}{C_{0}}=1-\frac{1+\left(\frac{Q C_{0} t}{q_{Y A N} m}\right)^{a}}{\text { Yan }}$ \\
Wolborska & $\frac{C}{C_{0}}=\exp \left(\frac{\beta C_{0}}{N_{0}} t-\frac{\beta Z}{u}\right)$ \\
\hline
\end{tabular}

Fonte: Adaptado Canteli (2013).

Também foram calculados o tempo útil do leito até o ponto de ruptura, o tempo total de saturação, a altura útil, a zona de transferência de massa e a máxima capacidade de adsorção do leito, de acordo com as Equações de 3 a 7, respectivamente (ZANG et al., 2011).

$t_{u}=\int_{0}^{t_{b}}\left(1-\frac{C_{t}}{C_{0}}\right) d t$

$$
\begin{aligned}
& t_{t}=\int_{0}^{\infty}\left(1-\frac{C_{t}}{C_{0}}\right) \\
& H_{u}=\frac{t_{u}}{t_{t}} H_{t} \\
& Z T M=H_{t}-H_{U} \\
& q_{U}=\frac{C_{0} Q}{1000 m} \int_{0}^{t_{s}}\left(1-\frac{C_{t}}{C_{0}}\right) d t
\end{aligned}
$$

\section{RESULTADOS E DISCUSSÕES}

\subsection{Isoterma de Adsorção}

A partir do procedimento explicado, a isoterma de adsorção foi construída, e os dados foram ajustados aos modelos, como mostra a Figura 2 e os resultados da Tabela 5.

Figura 2 - Isoterma e ajuste dos Modelos.

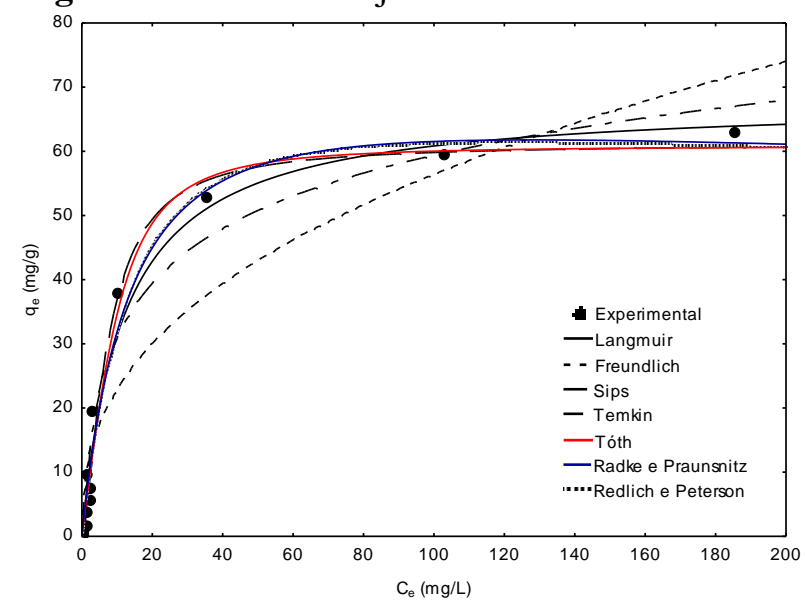

Fonte: Da Autora (2015).

A isoterma experimental apresentou um comportamento extremamente favorável, indicando que o adsorvente escolhido obteve um ótimo desempenho, e que quanto maior a concentração inicial maior foi o valor de adsorção máxima obtido.

Além disso, observou-se que a remoção do AM foi maior em concentrações mais baixas, atingindo $97,03 \%$ para a concentração de $50 \mathrm{mg} / \mathrm{L}$. Esse comportamento era esperado, já que a relação entre os sítios ativos do adsorvente e o adsorbato é maior, enquanto que para concentrações mais altas os sítios ativos são saturados mais rapidamente. 
Tabela 5 - Resultado do ajuste aos modelos.

\begin{tabular}{llrc}
\hline Modelos & Parâmetros & $\begin{array}{r}\text { Desvio } \\
\text { padrão }\end{array}$ & \multicolumn{1}{c}{$\mathbf{R}^{2}$} \\
\hline Langmuir & \multicolumn{2}{l}{$q_{\text {máx }}=67,979<3,8609$} & 0,9690 \\
& $b_{L}=0,0852$ & 0,0171 & \\
Freundlich & $k_{F}=9,2926$ & 2,6861 & 0,8539 \\
& $n=0,3917$ & 0,0640 & \\
Sips & $q_{\text {máx }}=61,175$ & 2,7500 & 0,9824 \\
& $k_{S}=0,05190$ & 0,0161 & \\
Temkin & $n_{S}=1,4662$ & 0,2026 & \\
& $\beta=12,4091$ & 0,8568 & 0,9544 \\
Tóth & $k_{T e}=1,1978$ & 0,2108 & \\
& $q_{\text {máx }}=60,834$ & 3,3278 & 0,9777 \\
& $k_{T}=0,0072$ & 0,0162 & \\
Radke e & $n_{T}=1,8714$ & 0,7707 & \\
Praunitz & $q_{\text {máx }}=94,166<34,5157$ & 0,9722 \\
& $b_{\text {rad }}=0,0563$ & 0,0291 & \\
Redlich e & $n_{\text {rad }}=1,1386$ & 1,1387 & \\
Peterson & $k_{R P}=5,0342$ & 1,0274 & 0,9734 \\
& $\alpha_{R P}=0,0433$ & 0,0296 & \\
\hline & $g=1,1105$ & 0,1047 & \\
\hline
\end{tabular}

Fonte: Da Autora (2015).

Analisando o coeficiente de correlação para os modelos estudados, observa-se que Freundlich apresentou o pior desempenho na correlação dos dados. Considerando o maior valor do coeficiente de correlação, o processo de adsorção de $\mathrm{AM}$ utilizando $\mathrm{BC}$ tem a tendência de seguir o modelo de Sips, o que pode ser ressaltado observando a melhor adequação através da Figura 2. Silva e Oliveira (2012), ao estudarem a adsorção do AM em bagaço de cana, encontraram o mesmo modelo de isoterma (Sips) com os seguintes parâmetros: $q_{\text {máx }}$ de $36,086 \mathrm{mg} / \mathrm{g}, K_{S}$ de 0,132 $\mathrm{L} / \mathrm{mg}$ e $n_{S}$ de 1,303 . O valor de $q_{\text {máx }}$ deste trabalho foi superior, indicando a maior eficiência de remoção deste bagaço.

\subsection{Cinética de Adsorção}

As Figuras 3 e 4 apresentam os resultados da cinética de adsorção, por meio da relação da quantidade adsorvida $q_{t}(\mathrm{mg} / \mathrm{g}) \mathrm{com}$ o tempo $t$ (min); enquanto a Tabela 5 mostra os valores dos parâmetros dos modelos cinéticos ajustados aos dados experimentais.

Figura 3 - Cinética de Adsorção Experimental Ajustada aos Modelos Cinéticos - $50 \mathrm{mg} / \mathrm{L}$.

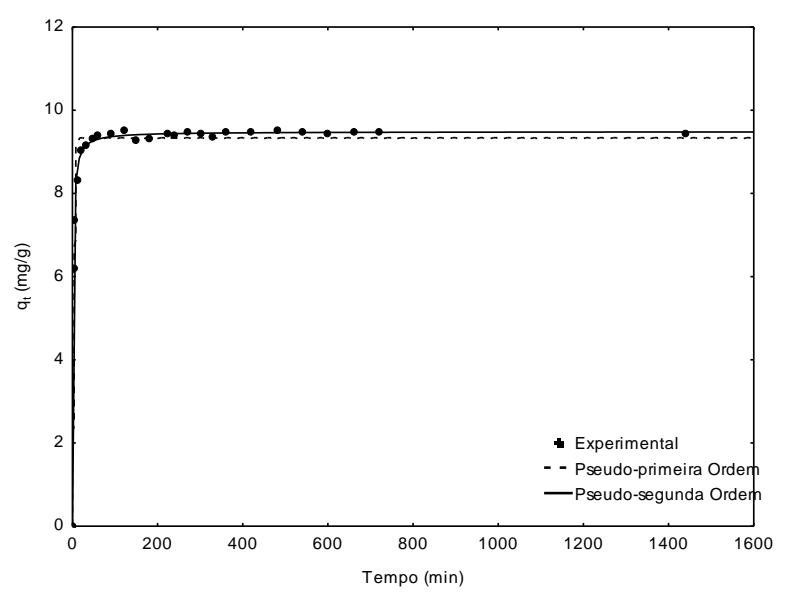

Fonte: Da Autora (2015).

Tabela 5 - Parâmetros dos Modelos de Cinética de Adsorção: concentrações iniciais de 50 e 200 mg/L.

\begin{tabular}{|c|c|c|c|c|c|c|}
\hline \multirow[b]{2}{*}{ Modelos } & \multicolumn{3}{|c|}{$\mathrm{C}_{0}=50 \mathrm{mg} / \mathrm{L}$} & \multicolumn{3}{|c|}{$\mathrm{C}_{0}=200 \mathrm{mg} / \mathrm{L}$} \\
\hline & Parâmetros & $\begin{array}{l}\text { Desvio } \\
\text { padrão }\end{array}$ & $\mathbf{R}^{2}$ & Parâmetros & $\begin{array}{l}\text { Desvio } \\
\text { padrão }\end{array}$ & $\mathbf{R}^{2}$ \\
\hline \multirow{2}{*}{$\begin{array}{c}\text { Pseudo } \\
1^{\text {a }} \text { ordem }\end{array}$} & $q_{e 1}=9,3411$ & 0,0724 & \multirow{2}{*}{0,9719} & $q_{e 1}=33,3625$ & 0,7338 & \multirow{2}{*}{0,8838} \\
\hline & $k_{1}=0,4392$ & 0,0369 & & $k_{1}=0,1002$ & 0,0167 & \\
\hline \multirow{2}{*}{$\begin{array}{c}\text { Pseudo } \\
2^{\mathrm{a}} \text { ordem }\end{array}$} & $q_{e 2}=9,4849$ & 0,0039 & \multirow{2}{*}{0,9970} & $q_{e 2}=34,8473$ & 0,0006 & \multirow{2}{*}{0,9586} \\
\hline & $k_{2}=0,0901$ & 0,0253 & & $k_{2}=0,0043$ & 0,5070 & \\
\hline
\end{tabular}

Fonte: Da Autora (2015). 
Figura 4 - Cinética de Adsorção Experimental Ajustada aos Modelos Cinéticos - 200 mg/L.

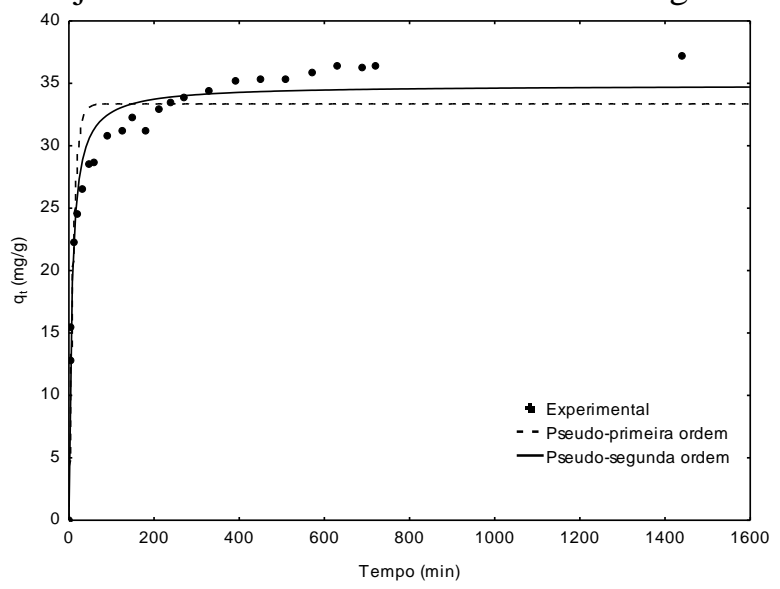

Fonte: Da Autora (2015).

Observou-se que o equilíbrio foi atingido rapidamente, em torno de 210 minutos para a concentração inicial de $50 \mathrm{mg} / \mathrm{L}$ e 330 minutos para a de $200 \mathrm{mg} / \mathrm{L}$, o que viabiliza tal processo em escala industrial. Essa diferença no tempo para atingir o equilíbrio, entre as concentrações, é devido ao processo de adsorção acontecer mais facilmente em concentrações menores. Em concentrações maiores, a transferência de massa acontece mais lentamente devido ao maior número de moléculas do corante presente na solução disputando a mesma quantidade de sítios ativos.

A partir dos resultados, verificou que o processo segue o modelo de Pseudo-segunda ordem, o qual foi o que melhor correlacionou os dados para ambas as concentrações.

Silva (2005) obteve um tempo de equilíbrio de aproximadamente 25 minutos, para o processo de adsorção de AM em serragem de Pinnus elliotti, com uma concentração inicial de $400 \mathrm{mg} / \mathrm{L}$. No trabalho, foi feito apenas o ajuste para o modelo de Pseudo $1^{\mathrm{a}}$ ordem, o qual apresentou uma representação eficiente dos dados, com $\mathrm{R}^{2}$ igual a 0,998 . Os parâmetros obtidos foram $q_{e}=239,93 \mathrm{mg} / \mathrm{g}$ e $k_{1}=641 \mathrm{~min}^{-1}$, os quais confirmam a rapidez com que o processo é realizado e a elevada eficiência de capacidade de adsorção desse material.

\subsection{Adsorção em Leito Fixo.}

Visto que a completa saturação do leito não foi alcançada $\left(C / C_{0}=1\right)$, a análise dos resultados foi feita, primeiramente ajustandose os dados aos modelos matemáticos, para que a partir desses fosse possível fazer o cálculo das outras características do leito fixo, a partir das Equações 3 a 7. Dessa maneira, os resultados dos ajuste dos modelos estão apresentados na Tabela 6 e na Figura 5.

Tabela 6 - Parâmetros dos modelos de Curva de Ruptura: (a) Condição 1; (b) Condição 2 e (c) Condição 3.

\begin{tabular}{|c|c|c|c|}
\hline Modelos & Parâmetros & $\begin{array}{l}\text { Desvio } \\
\text { Padrão }\end{array}$ & $\mathbf{R}^{2}$ \\
\hline \multicolumn{4}{|c|}{ Condição 1} \\
\hline Thomas & $\begin{array}{l}q_{m a ́ x}=40,7862 \\
k_{T h}=0,2320\end{array}$ & $\begin{array}{l}0,0094 \\
0,4042 \\
\end{array}$ & 0,9454 \\
\hline $\begin{array}{l}\text { Yoon \& } \\
\text { Nelson }\end{array}$ & $\begin{array}{l}q_{Y N}=40,7870 \\
k_{Y N}=0,0116\end{array}$ & $\begin{array}{l}2,2523 \\
0,0008\end{array}$ & 94 \\
\hline Yan & $\begin{array}{l}q_{Y A N}=40,3375 \\
a=4,0734\end{array}$ & $\begin{array}{l}0,3167 \\
0,1176\end{array}$ & 0,9756 \\
\hline Wolborska & $\begin{array}{l}\beta=1,1248 \\
N_{0}=8,2552\end{array}$ & & 0879 \\
\hline \multicolumn{4}{|c|}{ Condição 2} \\
\hline Thomas & $\begin{array}{r}q_{T h}=85, \\
k_{T h}=0\end{array}$ & & 0,9829 \\
\hline $\begin{array}{l}\text { Yoon \& } \\
\text { Nelson }\end{array}$ & $\begin{array}{r}q_{Y N}=85,7681 \\
k_{Y N}=0,0260\end{array}$ & $\begin{array}{l}2,2523 \\
0,0008 \\
\end{array}$ & 0,9829 \\
\hline Yan & $\begin{array}{l}q_{Y A N}=86,8428 \\
a=11,4560\end{array}$ & $\begin{array}{l}0,4606 \\
0,3864\end{array}$ & 0,9823 \\
\hline Wolborska & $\begin{array}{l}\beta=5,6072 \\
N_{0}=49,5317\end{array}$ & $\begin{array}{l}0,1486 \\
0,2389 \\
\end{array}$ & 0,9838 \\
\hline \multicolumn{4}{|c|}{ Condição 3} \\
\hline Thomas & $\begin{array}{r}q_{T h}=47,4464 \\
k_{T h}=0,4757\end{array}$ & $\begin{array}{l}0,0042 \\
0,3729\end{array}$ & 0,9697 \\
\hline $\begin{array}{l}\text { Yoon \& } \\
\text { Nelson }\end{array}$ & $\begin{array}{c}q_{Y N}=47,446 \\
k_{Y N}=0,0951\end{array}$ & $\begin{array}{l}0,6530 \\
0,0062\end{array}$ & 0,9697 \\
\hline Yan & $\begin{array}{l}q_{Y A N}=47,5174 \\
a=10,6589\end{array}$ & $\begin{array}{l}0,2514 \\
0,6474\end{array}$ & 53 \\
\hline Wolborska & $\begin{array}{l}\beta=2,3640 \\
N_{0}=3,6428\end{array}$ & $\begin{array}{l}0,1692 \\
0,0364\end{array}$ & 0,9511 \\
\hline
\end{tabular}

Fonte: Da Autora (2015). 
Figura 5 - Ajuste dos Modelos às Curvas de Ruptura: (a) Condição 1; (b) Condição 2 e (c) Condição 3.

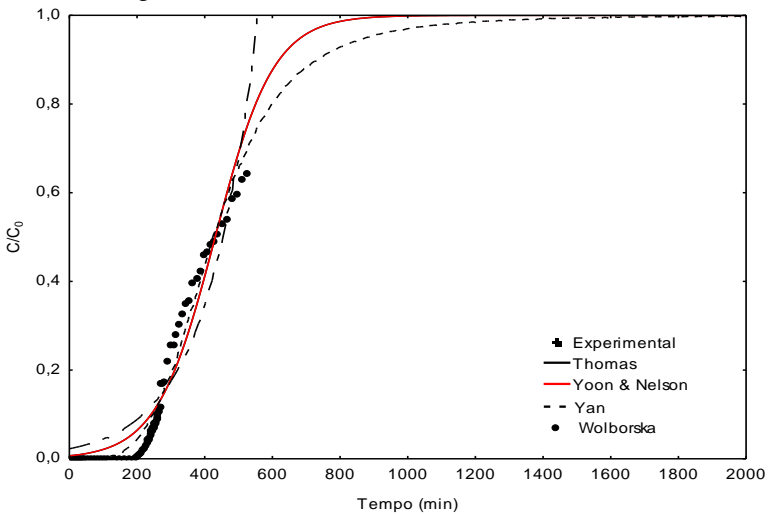

(a)

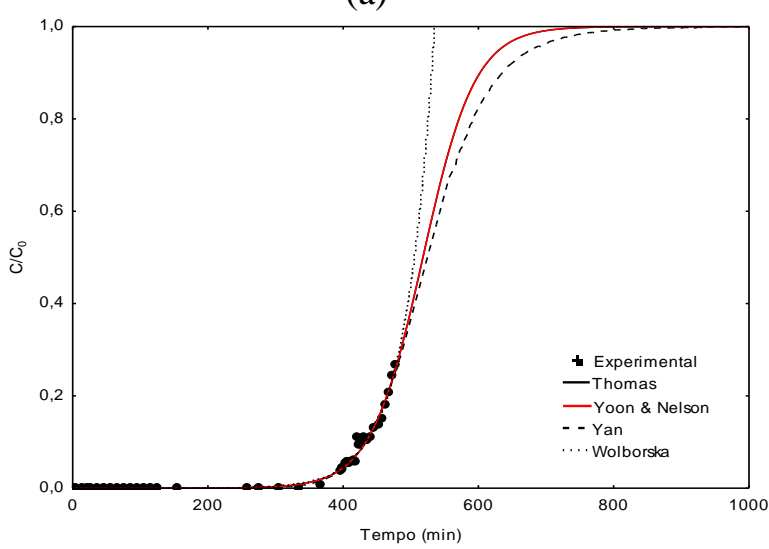

(b)

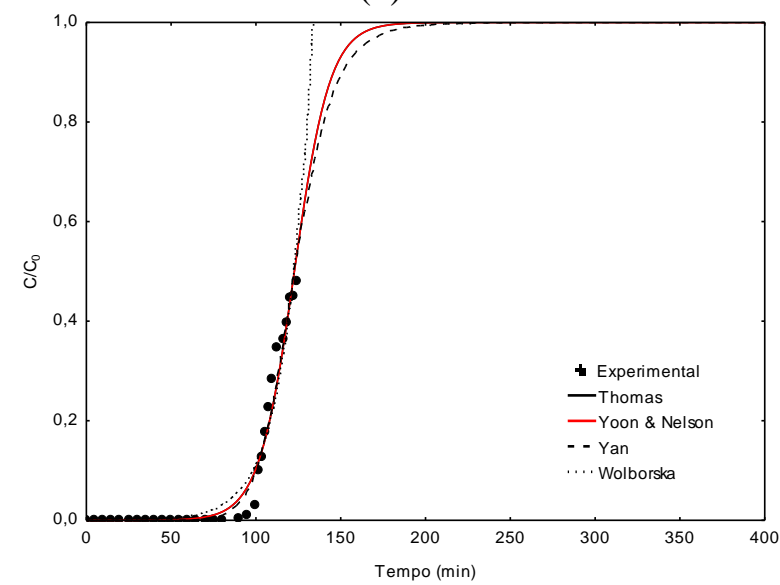

(c)

Fonte: Da Autora (2015).

O modelo de Thomas e o modelo de Yoon \& Nelson apresentaram valores exatamente iguais para o $\mathrm{R}^{2}$ em todas as condições estudadas, o que ocorreu devido às equações desses modelos serem análogas matematicamente.

O modelo de Yan foi o que melhor ajustou os dados para a maioria das condições (1 e 3), apresentando altos valores de $\mathrm{R}^{2}$. Por fim, o modelo de Wolborska se apresentou como o melhor ajuste apenas para a segunda condição, no entanto, Zangh et al. (2011) explica que esse modelo é válido apenas para baixas concentrações, no caso o último ponto experimental ficou em torno de $30 \%$ da concentração inicial.

A partir dos resultados, observou-se que todos os ajustes foram satisfatórios, mesmo sem o término do experimento.

Os outros parâmetros característicos do leito fixo estão apresentados na Tabela 7. Devido o modelo de Wolborska não permitir determinar o tempo de saturação, foi utilizado o modelo de Yoon \& Nelson para os cálculos da condição 2 .

Tabela 7 - Parâmetros Característicos do Leito Fixo.

\begin{tabular}{llll}
\hline Parâmetros & $\begin{array}{c}\text { Condição } \\
\mathbf{1}\end{array}$ & $\begin{array}{c}\text { Condição } \\
\mathbf{2}\end{array}$ & $\begin{array}{c}\text { Condição } \\
\mathbf{3}\end{array}$ \\
\hline$H_{t}(\mathrm{~cm})$ & 40 & 40 & 40 \\
$t_{\text {sat }}(\mathrm{min})$ & 474,33 & 518,47 & 122,07 \\
$t_{U}(\mathrm{~min})$ & 116,09 & 400,49 & 114,40 \\
$\%$ útil & 24,47 & 77,24 & 93,17 \\
$H_{U}(\mathrm{~cm})$ & 9,79 & 30,89 & 37,48 \\
$Z T M(\mathrm{~cm})$ & 30,21 & 9,10 & 2,52 \\
$q_{U}\left(\frac{\mathrm{mg}}{\mathrm{g}}\right)$ & 45,36 & 85,86 & 47,40 \\
\hline
\end{tabular}

Fonte: Da Autora (2015).

A eficiência de um leito fixo pode ser pode ser facilmente observada através da porcentagem útil do equipamento e/ou da ZTM. Um alto valor de porcentagem útil do leito acarreta em um baixo valor de ZTM. Nota-se que a para a Condição 1, o valor obtido para a ZTM foi muito alto, enquanto que para as outras duas condições o resultado foi mais 
satisfatório. Um valor alto representa uma pequena transferência de massa, a qual depende da vazão de alimentação e da concentração inicial da solução. De posse dos resultados, observou-se que uma concentração inicial maior, favoreceu a transferência de massa, uma vez que os melhores resultados foram para a terceira condição, na qual foi empregada uma vazão de alimentação próxima a da primeira condição.

Outro parâmetro importante é máxima capacidade de adsorção do leito, e para esse todas as condições apresentaram altos valores, indicando o potencial desse processo, contudo verificou-se que com o aumento da vazão de alimentação a capacidade máxima de adsorção do leito diminuiu.

Se torna importante pontuar também que o sistema conseguiu filtrar a solução por um longo período de tempo, de 100 até 418 minutos de operação, considerando a concentração de saída com valores menores que $15 \mathrm{mg} \mathrm{PT}-\mathrm{Co} / \mathrm{L}$, o qual corresponde ao valor de potabilidade da água (MINISTÉRIO DO MEIO AMBIENTE, 2006). Portanto, a realização de um scale-up desse processo pode ser vantajosa, por se tratar de um sistema contínuo e apresentar a capacidade de tratar grandes volumes de efluentes.

\section{CONCLUSÕES}

O presente trabalho estudou a capacidade do BC em adsorver o corante AM, a fim de removê-lo de soluções aquosas. Através do levantamento de dados experimentais foram obtidas as isotermas de equilíbrio, para avaliar o desempenho do adsorvente, a cinética do processo determinando o tempo necessário para chegar ao equilíbrio, e a dinâmica de adsorção em uma coluna de leito fixo.

O BC apresentou um elevado potencial para a adsorção do AM, alcançando $97,03 \%$ de remoção para a concentração de $50 \mathrm{mg} / \mathrm{L}$, $\mathrm{q}_{\mathrm{e}}$ de $63,93 \mathrm{mg} / \mathrm{g}$ para $500 \mathrm{mg} / \mathrm{L}$, e se adequando ao tipo de isoterma extremamente favorável, que pode ser representada pelo Modelo de Sips. Já a cinética de adsorção segue o modelo de pseudo-segunda ordem.

Em relação as curvas de ruptura obtidas, dentre as três condições observadas, a terceira apresentou melhores resultados, uma vez que a porcentagem útil do leito foi a maior $\mathrm{e}$ consequentemente menor valor de ZTM, indicando uma baixa resistência difusional. Foi possível concluir também com o aumento da vazão de alimentação a capacidade máxima de adsorção do leito diminui.

O modelo de Yan foi o que melhor ajustou os dados para a maioria das condições, apresentando valores de parâmetros coerentes com os obtidos experimentais. Já o modelo de Wolborska se apresentou como o melhor ajuste apenas para a segunda condição, no entanto, esse modelo é válido apenas para baixas concentrações, como era o caso da segunda condição.

\section{NOMENCLATURA}

$q_{e} \quad$ Quantidade de adsorbato adsorvido pelo adsorvente no equilíbrio $(\mathrm{mg} / \mathrm{g})$;

$b_{L} \quad$ Constante de afinidade de adsorção de Langmuir (L/mg);

$C_{e} \quad$ Concentração do equilíbrio (mg/L);

$k_{F} \quad$ Constante de Freundlich $(\mathrm{L} / \mathrm{mg})$;

$q_{\text {máx }}$ Capacidade máxima de adsorção do material $(\mathrm{mg} / \mathrm{g})$;

$n \quad$ Expoente de Freundlich;

$k_{S} \quad$ Constante de Sips (L/mg);

$n_{S} \quad$ Expoente de Sips;

$\beta \quad$ Parâmetro de Temkin;

$k_{T e} \quad$ Constante de ligação de equilíbrio (L/mg);

$k_{T} \quad$ Parâmetro de Tóth (L/mg);

$n_{T} \quad$ Constante específica de Tóth;

$b_{\text {rad }}$ Constante da isoterma de Radke e Praunsnitz (L/mg);

$n_{\text {rad }}$ Constante de Radke e Praunsnitz;

$k_{R P} \quad$ Constante de Redlich e Peterson (L/g);

$\alpha_{R P} \quad$ Parâmetro de Redlich e Peterson (mg/L);

$g \quad$ Expoente de Redlich e Peterson; 


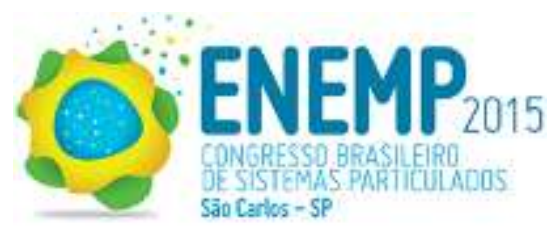

$q_{t} \quad$ Quantidade de adsorbato adsorvida no tempo $\mathrm{t}(\mathrm{mg} / \mathrm{g})$;

$q_{e 1} \quad$ Capacidade de adsorção no equilíbrio de Pseudo-primeira ordem $(\mathrm{mg} / \mathrm{g})$;

$q_{e 2} \quad$ Capacidade de adsorção no equilíbrio de Pseudo-segunda ordem (mg/g);

$k_{1} \quad$ Constante da taxa de adsorção de Pseudo-primeira ordem (mg/g)

$k_{2} \quad$ Constante da taxa de adsorção de Pseudo-segunda ordem (mg/g);

$t_{U} \quad$ Tempo útil do leito ( $\left.\mathrm{min}\right)$;

$t_{t} \quad$ Tempo de saturação do leito (min);

$C_{0} \quad$ Concentração inicial (mg/L);

$t_{b} \quad$ Tempo de ruptura do leito(min);

$H_{U} \quad$ Altura útil do leito $(\mathrm{cm})$;

$H_{t} \quad$ Altura total do leito $(\mathrm{cm})$;

ZTM Zona de transferência de massa $(\mathrm{cm})$;

$q_{U} \quad$ Capacidade máxima de adsorção do leito (mg/g);

$m \quad$ Massa de adsorvente;

$Q \quad$ Vazão de alimentação;

$t \quad$ Tempo (min);

$u \quad$ Velocidade superficial $(\mathrm{cm} / \mathrm{min})$;

C Concentração da solução à saída do leito $(\mathrm{mg} / \mathrm{L})$;

$K_{T h} \quad$ Constante cinética de Thomas (ml/min.mg);

$q_{T h} \quad$ Capacidade máxima de adsorção (mg/g);

$K_{Y N} \quad$ Constante cinética de Yoon \& Nelson $\left(\mathrm{min}^{-1}\right)$;

$q_{Y N} \quad$ Capacidade máxima de adsorção de Yoon \& Nelson (mg);

$q_{Y A N} \quad$ Capacidade de adsorção do leito $(\mathrm{mg} / \mathrm{g})$;

$a \quad$ Expoente de Yan;

$\beta \quad$ Coeficiente cinético da transferência de massa externa $\left(\mathrm{min}^{-1}\right)$

$N_{0} \quad$ Capacidade de saturação do adsorvente por unidade de volume do leito fixo (mg/L)

$Z \quad$ Altura do empacotamento (cm);

$V \quad$ Volume de solução (L).

\section{REFERÊNCIAS}

CANTELI, A. M. D. Recuperação do Aroma de Café, Benzaldeído, em Coluna de Adsorção Utilizando Carvão Ativado. Tese (Mestrado em Engenharia de Alimentos) - Universidade Federal do Paraná, 2013.
XXXVII ENEMP

18 a 21 de Outubro de 2015

Universidade Federal de São Carlos
COLOMBO, A. Biossorção dos Íons Cádmio e Chumbo pela Casca de Soja. Tese (Mestrado em Engenharia Química) - Universidade Estadual do Oeste do Paraná, Toledo, 2013.

CONAMA. Conselho Nacional do Meio Ambiente. Disponível em: http://www.mma.gov.br/port/conama/res/res05/res 35705.pdf. Acesso em: 17 mai. 2015.

GUARATINI C.C. I. ; ZANONI M. V. B. Corantes têxteis. Química Nova, v. 23, p. 71-78, 2000.

McCABE, W. L., SMITH, J. C., HARRIOT, P. Unit Operations of Chemical Enginnering. McGraw Hill Education, 7th ed., 2014.

MAPA-Ministério da Agricultura. Disponível em:http://www.agricultura.gov.br/vegetal/culturas /cana-de-acucar. Acesso em: 20 fev. 2015.

OLIVEIRA, S. P. Adsorção do Corante Azul de Metileno em Caulinita Natural e Intercalada com Acetato de Potássio Provenientes da Região de Bom Jardim de Goiás - GO. Tese (Mestrado em Geociências) - Universidade Federal do Mato Grosso, Cuiabá, 2012.

\section{SCHIMELL, D. Adsorção dos Corantes Reativos} Azul 5g e Azul Turquesa QG em

Carvão Ativado Comercial. Tese (Mestrado em Engenharia Química) - Universidade Estadual do Oeste do Paraná, Toledo, 2008.

SILVA, A. T. Influência da Temperatura na Adsorção do Corante Azul de Metileno Utilizando Serragem de Pinus Elliottii como um Adsorvente Alternativo: Um Modelo para o Tratamento de Efluentes Têxteis. Universidade Federal de Santa Catarina, Florianópolis, 2005.

SILVA, W. L. L.; OLIVEIRA, S. P. Modificação das Características de Adsorção do Bagaço de Cana para Remoção de Azul de Metileno de Soluções Aquosas. Scientia Plena, v.8, n. 9, 2012.

ZHANG, W.; L. DONG, H. YAN, H. LI, Z. JIANG, X. KAN, H. YANG, A. LI, R. Cheng. "Removal of Methylene Blue From Aqueous Solutions by Straw Based Adsorbent in a Fixedbed Column". Chemical Enginnering Journal, v. 173, p 429-433, 2011. 